\title{
Non-stenting strategy is not inferior to stent implantation in patients with acute ST-segment elevated myocardial infarction and high thrombus burden and intermediate stenotic culprit lesion
}

\author{
Demou Luo ${ }^{1}$, Xing Yang ${ }^{2}$, Xiangming $\mathrm{Hu}^{3}$, Jixin Liu ${ }^{4}$, Chenyang Wang ${ }^{5}$, Jingguang $\mathrm{Ye}^{2}$, Xiaosheng Guo ${ }^{2}$, \\ Shenghui $\mathrm{Xu}^{2}$, Boyu Sun ${ }^{2}$, Yongdong Liu ${ }^{5}$, Shuo Sun ${ }^{5}$, Yingling Zhou ${ }^{5}$, Zheng Huang', Youti Zhang ${ }^{4}$, \\ Haojian Dong
}

\begin{abstract}
${ }^{1}$ Department of Cardiology, the First Affiliated Hospital of Guangzhou Medical University, Guangzhou, China; ${ }^{2}$ Department of Interventional Treatment, Guangdong Provincial People’s Hospital Zhuhai Hospital, Zhuhai, China; ${ }^{3}$ The Second School of Clinical Medicine, Southern Medical University, Guangzhou, China; ${ }^{4}$ Department of Cardiology, Guangdong Provincial Jiexi People's Hospital, Jiexi, China; ${ }^{5}$ Guangdong Cardiovascular Institute, Guangdong Provincial People's Hospital, Guangdong Academy of Medical Sciences, Guangzhou, China

Contributions: (I) Conception and design: H Dong, Y Zhou, Z Huang, Y Zhang; (II) Administrative support: D Luo, S Sun, X Hu; (III) Provision of study materials or patients: X Yang, B Sun, C Wang, Y Liu; (IV) Collection and assembly of data: J Ye, X Guo, S Xu, J Liu; (V) Data analysis and interpretation: Y Zhou, H Dong, D Luo; (VI) Manuscript writing: All authors; (VII) Final approval of manuscript: All authors.

Correspondence to: Haojian Dong. Guangdong Cardiovascular Institute, Guangdong Provincial People's Hospital, Guangdong Academy of Medical Sciences, 96 Dongchuan Road, Guangzhou 510080, China. Email: donghaojian@sina.com; Youti Zhang. Department of Cardiology, Guangdong Provincial Jiexi People's Hospital, 7 Dangxiao Road, Jiexi 515400, China. Email: 1131516612@qq.com.
\end{abstract}

\begin{abstract}
Background According to European Society of Cardiology (ESC) as well as American College of Cardiology/American Heart Association (ACC/AHA) guidelines, primary stenting is recommended for patients with acute ST-segment elevation myocardial infarction (STEMI); however, in-stent thrombosis is a life-threatening early adverse event that could lead to acute myocardial infarction (AMI) or even cardiac death. On the other hand, in-stent restenosis is a late adverse event that could result in recurrent readmission and revascularization. We compared a non-stenting (NS) strategy to a stenting (S) strategy in terms of incidence of major adverse cardiac events (MACEs) for patients with acute STEMI and high thrombus burden.
\end{abstract}

Methods: We performed a post hoc analysis of our prior multicenter, prospective cohort study (ChiCTR1800019923) among 51 eligible patients with acute STEMI and high thrombus burden. All participants received percutaneous coronary intervention (PCI) with a deferred-stenting strategy (second procedure performed within 48-72 h after primary PCI). Either NS or S strategies were carried out among patients. Primary outcomes were follow-ups of MACEs at 1, 3, 6, and 12 months. Intravenous ultrasound (IVUS) and quantitative flow ratio (QFR) evaluation were performed.

Results: In our post hoc analysis of 51 patients (21 with NS and 30 with S), baseline clinical and interventional characteristics were well matched between the 2 groups, to the exception of culprit lesion length. Incidence of MACEs was not significantly different between the 2 strategies in-hospital $(\mathrm{P}=0.56)$ and in follow-ups at 1 ( $\mathrm{P}=0.41), 3$ (free of events), $6(\mathrm{P}=0.71)$, and 12 ( $\mathrm{P}=0.68)$ months. Culprit lesions of NS tended to be "lowrisk" [minimum lumen area (MLA) $4.27 \pm 1.02$ vs. $3.80 \pm 1.32 \mathrm{~mm}^{2}, \mathrm{P}=0.36$ ] and plaque burden $(70.79 \% \pm 6.46 \%$ vs. $76.97 \% \pm 6.76 \%, \mathrm{P}=0.03$ ) when compared with culprit lesions of $\mathrm{S}$ in IVUS evaluation. Evaluation of QFR showed more sufficient physiological reperfusion improvement with NS than with $\mathrm{S}$ [two-dimensional (2D) QFR: $0.85 \pm 0.09$ vs. $0.79 \pm 0.13, \mathrm{P}=0.10$ and $3 \mathrm{D}$ QFR: $0.86 \pm 0.08$ vs. $0.78 \pm 0.15, \mathrm{P}=0.02]$.

Conclusions: The NS strategy did not increase MACEs in-hospital and at 1, 3, 6, and 12 months. The NS can be a safe option when meeting certain criteria for patients with STEMI and a high thrombus burden.

\footnotetext{
Keywords: ST-segment elevation myocardial infarction (STEMI); deferred stenting; non-stenting; major adverse cardiac event (MACE); high thrombus burden
} 
Submitted Aug 16, 2021. Accepted for publication Oct 19, 2021.

doi: 10.21037/apm-21-2612

View this article at: https://dx.doi.org/10.21037/apm-21-2612

\section{Introduction}

Acute ST-segment elevation myocardial infarction (STEMI) is mostly caused by intra-lumen thrombus resulting from plaque rupture, plaque erosion, or calcified nodules $(1,2)$. Reperfusion of ischemic myocardium is the first priority, and primary percutaneous coronary intervention (pPCI) is a preferred recommendation to restore blood flow of an infarct-related artery (IRA), according to ESC as well as ACC/AHA guidelines. Primary stenting is an effective strategy (Class I, Level A) (3); however, the incidence of acute in-stent thrombolysis, early or late in-stent restenosis, as well as intravascular intimal hyperplasia remains (4). Previous studies have indicated that the percentage stenosis rate (about $35 \%$ ) of native plaques resulting in STEMI usually ranges from mild to moderate $(5,6)$. According to the findings of some studies, based on comprehensive therapy of optimized antiplatelet agents (anti-thrombus) and statin (plaque stabilized), a non-stenting (NS) strategy might be an alternative in some patients with STEMI but without severe residual stenosis $(7,8)$. Reports showed a higher incidence of restenosis with left anterior descending artery through non-stent strategy to patients with coronary artery disease (9). However, the pathophysiological mechanism of target lesions is different in STEMI with high thrombus burden. In our prospective study, we aimed to explore whether or not morphological and physiological examination [intravenous ultrasound (IVUS) and quantitative flow ratio (QFR)]-guided comprehensive therapy [dual-antiplatelet plus statins, glycoprotein IIb/ IIIa receptor antagonists, angiotensin converting enzyme inhibitors (ACEIs), or angiotensin receptor blockers (ARBs), as well as $\beta$-blockers] without stenting is inferior to a stenting (S) strategy in MACEs. We present the following article in accordance with the STROBE reporting checklist (available at https://dx.doi.org/10.21037/apm-21-2612).

\section{Methods}

\section{Study design and setting}

We performed subgroup analysis of our previous multicenter, open-label, prospective cohort study ("The outcomes in STEMI patients with high thrombus burden treated by deferred versus immediate stent implantation in primary percutaneous coronary intervention: a prospective cohort study", registered at www.chictr. org.cn, ChiCTR1800019923), which was conducted at 3 cardiovascular centers in South China (Guangdong Provincial People's Hospital, Guangzhou City; Guangdong Provincial People's Hospital Zhuhai Hospital, Zhuhai City; and Guangdong Provincial Jiexi People's Hospital, Jiexi City) from January 2018 to January 2020 (Figure 1).

Patients with a confirmed diagnosis of acute STEMI and indications for pPCI were invited to participate in our study. Comprehensive evidence-based drug therapy was routinely prescribed after the obtainment of informed consent. The pPCI was performed normally, while participants underwent deferred stent (DS) implantation or immediate stent (IS) implantation at the discretion of the operators (blinded to the trial) based on routine processing as well as contemporary guidelines. Participants who received a DS strategy, including NS or S, underwent a follow-up at 1, 3, 6, and 12 months. Data of quantitative coronary analysis (QCA) as well as IVUS during PCI were collected. The investigators performed no additional intervention during the entire study period. This research was approved by the Ethics Committee of Guangdong Provincial People's Hospital [GDREC2018346H(R2)]. All procedures performed in this study involving human participants were in accordance with the Declaration of Helsinki (as revised in 2013).

\section{Study population}

Patients who met the following inclusion criteria were included: (I) age $\geq 18$ years; (II) diagnosed with acute STEMI and had been recommended pPCI; (III) imaging manifestations of a high thrombus burden during surgery using angiography [tissue score (TS) $>2 ; 0=$ no thrombus; $1=$ haziness; $2=$ definite thrombus $<1 / 2$ vessel diameter; $3=$ definite thrombus $1 / 2$ to 2 vessel diameters; and $4=$ definite thrombus $>2$ vessel diameters; $5=$ total occlusion] (10); (IV) a thrombus in myocardial infarction (TIMI) flow grade of 2-3 was achieved after primary intervention before stent 


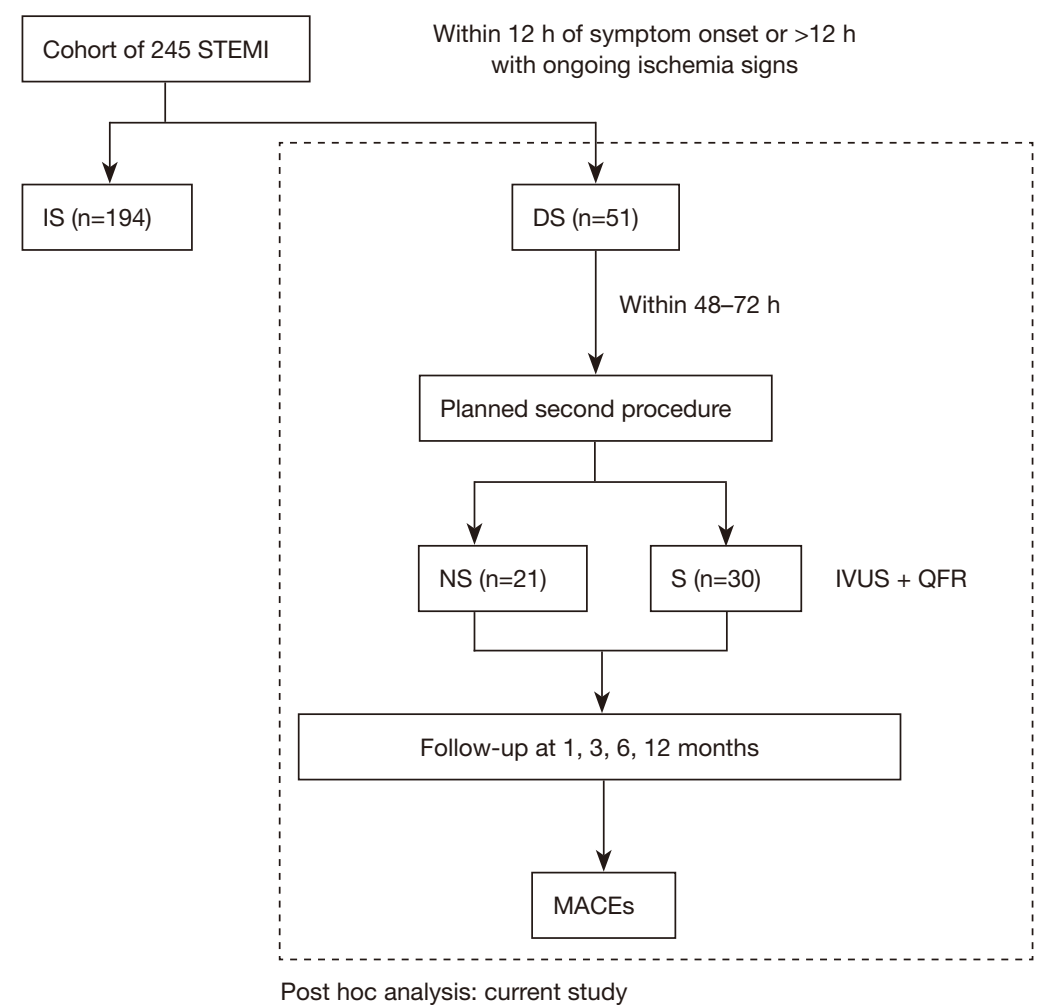

Figure 1 Flowchart of the current study. STEMI, ST-segment elevation myocardial infarction; IS, immediate stenting; DS, deferred stenting; IVUS, intravenous ultrasound; QFR, quantitative flow ratio; MACE, major adverse cardiac event.

implantation; and $(\mathrm{V})$ informed consent was provided.

Patients who met the following criteria were excluded: (I) cardiogenic shock; (II) IRA resulting from in-stent lesions or saphenous vein bypass graft abnormality; (III) dissection in an IRA requiring stent implantation; (IV) history of contrast allergy; (V) life expectancy $<12$ months; (VI) active bleeding; (VII) left main artery as the IRA; (VIII) left ventricular ejection fraction (LVEF) $<30 \%$; (IX) hemoglobin in plasma $<70 \mathrm{~g} / \mathrm{L}$; $(\mathrm{X})$ platelet count in plasma $<50 \times 10^{9} / \mathrm{L}$; and $(\mathrm{XI})$ poor compliance.

Participants were informed that they had the right to withdraw from the study at any time for any reason throughout the entire research process.

After recruitment, 51 patients with a DS strategy were included in the study, with 21 receiving NS and 30 receiving S. A total of 46 participants $(90.2 \%)$ underwent QCA evaluation, whereas $49.0 \%$ (25/51) were examined with IVUS after coronary angiography (CAG). Major adverse cardiac events (MACEs) were followed-up in the whole cohort.

\section{Interventional procedure, IVUS images, and QCA image acquisition}

We performed CAG using a radial or femoral approach in accordance with routine procedures stipulated by the Guangdong Provincial People's Hospital. The selection of the angiographic catheters, guidewires, guiding catheters, balloons, and the stent was at the discretion of the cardiologist. All participants were administered nitroglycerin $(100-200 \mu \mathrm{g})$ or glycoprotein (GP) IIb/ IIIa receptor antagonist $(10-20 \mathrm{~mL})$ intra-IRA unless contraindicated. Administration of GP IIb/IIIa receptor antagonist was continued intravenously at $0.1 \mu \mathrm{g} / \mathrm{kg} / \mathrm{min}$ for 24-48 h after surgery. Aspiration thrombectomy (using a Rebirth Pro ${ }^{\circledR}$ thrombus aspiration catheter; Goodman, Naka-ku, Nagoya, Japan) was performed at the discretion of the operator. Dual-antiplatelet therapy with ACEI/ARB, statins, and $\beta$-blockers was prescribed. A second CAG was performed at $48-72 \mathrm{~h}$ after the primary procedure, during which QCA was calculated on the basis of angiographic results and culprit lesion images acquired using IVUS 
(iLab ${ }^{\text {TM }}$ Polaris MultiModality Guidance System, H749ILAB220C270; Boston Scientific, San Jose, CA, USA) after CAG.

We defined $\geq 50 \%$ filling defect of the reference lumen diameter in epicardial arteries as significant stenosis, with QCA or IVUS evaluation. Thrombus score (TS), as well as the TIMI flow grade, was also measured after CAG.

Minimum diameter stenosis (\%), lesion length (mm), remodeling index (RI), minimum lumen diameter $(\mathrm{mm})$, plaque area $\left(\mathrm{mm}^{2}\right)$, plaque burden (\%), minimum lumen area stenosis rate (\%), plaque eccentricity index, and incidence of plaque rupture or calcified nodule (\%) were evaluated using IVUS. Eligible participants received off-line QFR evaluation by 2 experienced technicians, who were blinded to the study, in an independent core laboratory of Shanghai, China. Computation of QFR was performed using a prototype software (AngioPlus Core; Pulse Medical Imaging Technology, Shanghai, China), with the cutoff value for physiological significance as 0.80 (10).

\section{Outcomes}

The primary outcomes of the study were the incidence of MACEs, defined as a composite of recurrent acute myocardial infarction, cardiac death, all-cause mortality, acute heart failure-induced rehospitalization, target lesion revascularization, and major bleeding, during hospitalization or at the $1,3,6$, or 12 months postoperative follow-up.

The secondary outcomes were the IVUS and QFR evaluation of the culprit lesion to further analyze the pathological characteristics of the IRA and culprit plaque by obtaining angiographic results during the planned CAG 48-72 h after the primary operation.

\section{Statistical analysis}

We identified significant differences between the groups using the $t$-test or analysis of variance for normally distributed data, the Mann-Whitney U test or KruskalWallis $\mathrm{H}$ test for skewed distribution data, and the chisquared test or Fisher's exact test for categorical variables. The Kaplan-Meier method was used to describe and confirm the cumulative incidence of MACEs, and the log-rank test was conducted to compare groups. Cox proportional hazards regression models were used to evaluate the association of clinical or interventional parameters with the risk of MACEs. A 2-sided $\mathrm{P}$ value $<0.05$ was considered to indicate statistical significance.

\section{Results}

A total of 51 patients participated during the 2 years of recruitment (21 with NS and 30 with S, $41.2 \%$ vs. $58.8 \%$, respectively). The NS and S strategies were well matched between the groups in terms of baseline clinical and interventional characteristics, except for the length of the culprit lesion (Table 1). All participants in the NS or S group had complete follow-up at 1, 3, 6, and 12 months via telephone or outpatient services, and information of MACEs was recorded. The TIMI myocardial perfusion grade (TMPG) classification and imaging data calculated using a IVUS or QFR approach were collected.

\section{Primary outcomes}

A solitary patient $(3.3 \%)$ in the S group had in-hospital myocardial infarction (MI) versus none in the NS group $(\mathrm{P}=0.40)$. There were 2 major bleeding events $(9.5 \%)$ in the NS group, and no bleeding occurred in the $\mathrm{S}$ group $(\mathrm{P}=0.09)$.

There was 1 participant (4.8\%) in the NS group who had been re-admitted to the hospital owing to acute heart failure at 1-month-follow-up, with no significant difference compared with participants in the $\mathrm{S}$ group $(\mathrm{P}=0.21)$. No participants had any MACEs at the 3 months follow-up.

At the 6 months follow-up, 1 of 21 (4.8\%) participants in the NS group had non-IRA ischemic symptoms before receiving revascularization, with no significant difference as compared with the $\mathrm{S}$ group $(\mathrm{P}=0.41)$. Major bleeding (all gastrointestinal bleeding) was observed in 1 of 21 (4.8\%) participants in the NS group and 2 of $30(6.7 \%)$ participants in the $\mathrm{S}$ group, with no obvious difference between the groups $(\mathrm{P}=0.78)$. At the 12 months follow-up, no myocardial infarction, cardiac death, all-cause mortality, or heart failure-induced re-admission were observed among the participants. There was $1(4.8 \%)$ participant who had experienced IRA-related chest pain and had undergone PCI in the NS group, and 1 (3.3\%) participant had evidence of ischemia resulting from in-stent restenosis of the IRA, for which a revascularization was also performed $(\mathrm{P}=0.80)$. Major bleeding occurred in 2/21 (9.5\%) and 2/30 (6.7\%) participants with an NS strategy or S strategy (1 with urinary bleeding and 3 with gastrointestinal bleeding), with no significant difference $(\mathrm{P}=0.71)$ (Tables 2-6). 
Table 1 Comparison of patients' baseline clinical or interventional characteristics between NS and S strategies

\begin{tabular}{|c|c|c|c|}
\hline Characteristic & NS strategy group $(n=21)$ & S strategy group $(n=30)$ & $P$ value \\
\hline Gender (male) & $18(85.7)$ & $26(86.7)$ & 0.92 \\
\hline Hypertension & $10(47.6)$ & $17(56.7)$ & 0.52 \\
\hline Diabetes & $5(23.8)$ & 7 (23.3) & 0.97 \\
\hline CKD & $0(0.0)$ & $1(3.3)$ & 0.40 \\
\hline BMI, $\mathrm{kg} / \mathrm{m}^{2}$ & $24.7 \pm 2.6$ & $25.2 \pm 3.4$ & 0.55 \\
\hline LDLc (mmol/L) & $3.42 \pm 1.05$ & $3.11 \pm 1.01$ & 0.29 \\
\hline $\mathrm{TG}$ (mmol/L) & $1.63 \pm 1.12$ & $1.32 \pm 0.93$ & 0.29 \\
\hline Crea $(\mu \mathrm{mol} / \mathrm{L})$ & $78.22 \pm 25.72$ & $84.56 \pm 30.51$ & 0.44 \\
\hline Culprit vessel & & & 0.35 \\
\hline $\mathrm{RCA}$ & 7 (33.3) & $15(50)$ & \\
\hline LAD & $12(57.1)$ & $11(36.7)$ & \\
\hline LCX & $2(9.5)$ & $4(13.3)$ & \\
\hline Lesion length (mm) & $12.50 \pm 5.89$ & $17.48 \pm 8.76$ & 0.03 \\
\hline Stenosis (\%) & $49 \pm 26$ & $56 \pm 18$ & 0.24 \\
\hline \multicolumn{4}{|l|}{ No. of diseased arteries } \\
\hline TIMI 3 & $19(90.5)$ & $28(93.3)$ & \\
\hline
\end{tabular}

Data are shown as median (mean \pm SD) or $\mathrm{n}(\%)$. NS, non-stenting; S, stenting; CKD, chronic kidney disease; MI, myocardial infarction; BMI, body mass index; LDLc, low-density lipoprotein cholesterol; TG, triglycerides; HDLc, high-density lipoprotein cholesterol; UA, uric acid; Crea, creatinine; RCA, right coronary artery; LAD, left anterior descending artery; LCX, left circumflex artery; CAG, coronary angiography; TIMI, thrombolysis in myocardial infarction.

\section{Secondary outcomes}

\section{IVUS image endpoints}

In the post hoc analysis, blood flow of TIMI grade 2-3 in epicardial arteries was found among all participants in a planned second operation. In total, 10 of 21 (47.6\%) participants in the NS group and 15 of $30(50.0 \%)$ participants in the $\mathrm{S}$ group underwent IVUS examination during the planned second procedure. Information of morphological parameters was collected. After analysis, we found no differences between the NS and S groups in terms of reference lumen $(3.84 \pm 0.66$ vs. $3.95 \pm 0.83 \mathrm{~mm}$, respectively, $\mathrm{P}=0.72)$, minimal lumen diameter $(2.08 \pm 0.33$ vs. $2.01 \pm 0.35 \mathrm{~mm}$, respectively, $\mathrm{P}=0.61)$, minimal lumen diameter restenosis $(44.61 \% \pm 11.48 \%$ vs. $47.84 \% \pm 11.32 \%$, respectively, $\mathrm{P}=0.49$ ), minimum external elastic membrane cross-sectional area (EEM CSA; $15.25 \pm 4.60 v s$. 
Table 2 Primary outcomes of in-hospital MACEs postoperatively

\begin{tabular}{lccc}
\hline Outcome & NS strategy group & S strategy group & P value \\
\hline In-hospital MI & $0(0)$ & $1(3.3)$ & 0.40 \\
In-hospital CD & $0(0)$ & $0(0)$ & - \\
In-hospital ACM & $0(0)$ & $0(0)$ & - \\
In-hospital HF & $0(0)$ & $0(0)$ & - \\
In-hospital bleeding & $2(9.5)$ & $0(0)$ & 0.09 \\
Total MACEs & $2(9.5)$ & $1(3.3)$ & 0.56 \\
\hline
\end{tabular}

Data are shown as $\mathrm{n}(\%)$. Table format cited from previous study reference (11). MACE, major adverse cardiovascular event; NS, nonstenting; S, stenting; MI, myocardial infarction; CD, cardiac death; ACM, all-cause-mortality; HF, heart failure.

Table 3 Primary outcomes of MACEs at 1-month postoperative follow-up

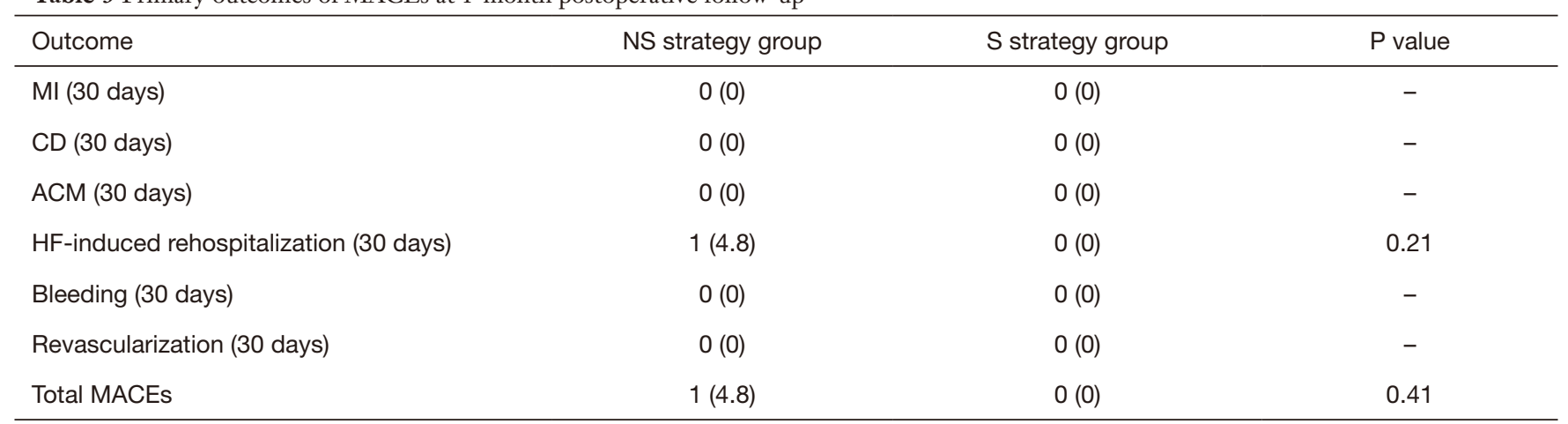

Data are shown as $\mathrm{n}$ (\%). Table format cited from previous study reference (11). MACE, major adverse cardiovascular event; NS, nonstenting; S, stenting; MI, myocardial infarction; CD, cardiac death; ACM, all-cause-mortality; HF, heart failure.

Table 4 Primary outcomes of MACEs at 3-month postoperative follow-up

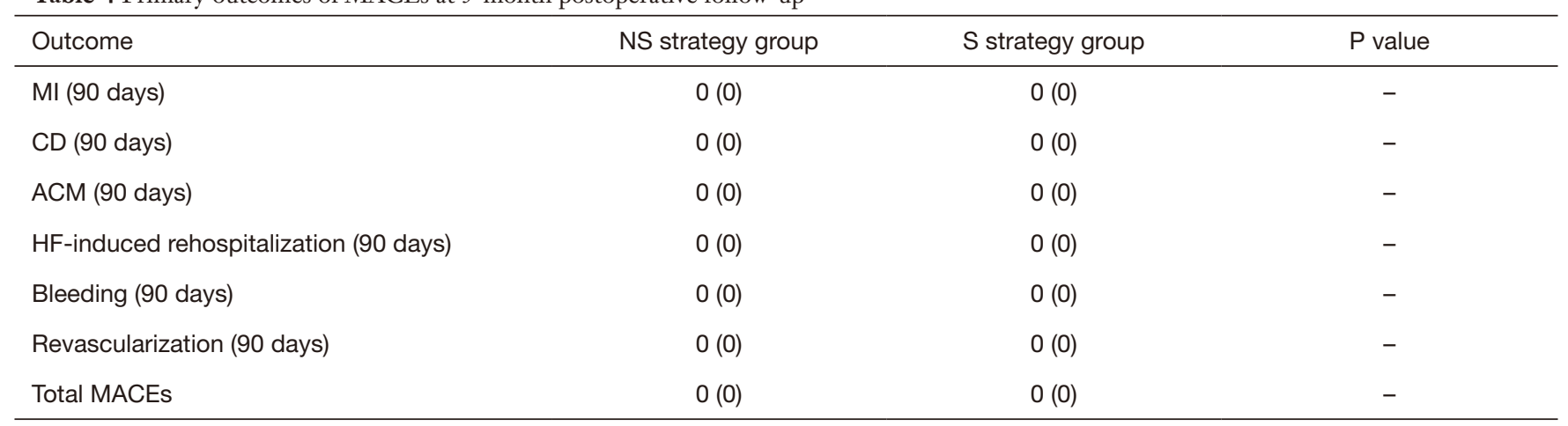

Data are shown as $\mathrm{n}$ (\%). Table format cited from previous study reference (11). MACE, major adverse cardiovascular event; NS, nonstenting; S, stenting; MI, myocardial infarction; CD, cardiac death; ACM, all-cause-mortality; HF, heart failure.

$17.05 \pm 5.27 \mathrm{~mm}^{2}$, respectively, $\left.\mathrm{P}=0.39\right)$, minimal lumen area (MLA; $4.27 \pm 1.02$ vs. $3.80 \pm 1.32 \mathrm{~mm}^{2}$, respectively, $\mathrm{P}=0.36)$, plaque area $\left(10.99 \pm 3.84\right.$ vs. $13.25 \pm 4.52 \mathrm{~mm}^{2}$, respectively, $\mathrm{P}=0.21)$, remodeling index $(1.06 \pm 0.20 v s$.
$1.07 \pm 0.19$, respectively, $\mathrm{P}=0.91)$, and plaque eccentricity index $(64.28 \% \pm 18.92 \%$ vs. $50.77 \% \pm 31.27 \%$, respectively, $\mathrm{P}=0.24)$. Among all anatomical information, the plaque burden in patients with an NS strategy was more 
Table 5 Primary outcomes of MACEs at 6-month postoperative follow-up

\begin{tabular}{|c|c|c|c|}
\hline Total & NS strategy group & S strategy group & $P$ value \\
\hline CD (6 months) & $0(0)$ & $0(0)$ & - \\
\hline ACM (6 months) & $0(0)$ & $0(0)$ & - \\
\hline HF-induced rehospitalization (6 months) & $0(0)$ & $0(0)$ & - \\
\hline Revascularization (6 months) & $1(4.8)$ & $0(0)$ & 0.41 \\
\hline Total MACEs & $2(9.5)$ & $2(6.7)$ & 0.71 \\
\hline
\end{tabular}

Data are shown as $\mathrm{n}$ (\%). Table format cited from previous study reference (11). MACE, major adverse cardiovascular event; NS, nonstenting; S, stenting; MI, myocardial infarction; CD, cardiac death; ACM, all-cause-mortality; HF, heart failure.

Table 6 Primary outcomes of MACEs at 12-month postoperative follow-up

\begin{tabular}{|c|c|c|c|}
\hline Total & NS strategy group & S strategy group & $P$ value \\
\hline CD (12 months) & $0(0)$ & $0(0)$ & - \\
\hline ACM (12 months) & $0(0)$ & $0(0)$ & - \\
\hline HF-induced rehospitalization (12 months) & $0(0)$ & $0(0)$ & - \\
\hline Revascularization (12 months) & $1(4.8)$ & $1(3.3)$ & 0.80 \\
\hline Total MACEs & $3(14.3)$ & $3(10.0)$ & 0.68 \\
\hline
\end{tabular}

Data are shown as $\mathrm{n}(\%)$. Table format cited from previous study reference (11). MACE, major adverse cardiovascular event; NS, nonstenting; S, stenting; MI, myocardial infarction; CD, cardiac death; ACM, all-cause-mortality; HF, heart failure.

severe than in those with an S strategy $(70.79 \pm 6.46$ vs. $76.97 \pm 6.76 \mathrm{~mm}$, respectively, $\mathrm{P}=0.03)$. Although the MLA stenosis in the NS group was $71.86 \% \pm 16.48 \%(<75 \%$ is a predictor of non-severe stenosis) and $82.50 \% \pm 17.56 \%$ ( $\geq 75 \%$, defined as severe stenosis) in the $\mathrm{S}$ group, no statistical difference was found between the 2 groups $(\mathrm{P}=0.14)$. Ascertainable plaque rupture was seen in 10 $(100 \%)$ participants in the NS group and in $12(80 \%)$ participants in the $\mathrm{S}$ group $(\mathrm{P}=0.13)$ (Table 7).

\section{QFR evaluation endpoints}

In total, 46 of 51 participants $(90.2 \%)$ were evaluated with QFR, with 17 of $21(90.0 \%)$ in the NS group and 29 of 30 (96.7\%) in the S group. The QFR was calculated in the last image of CAG in the NS group and in the last image of CAG before stent implantation in the $\mathrm{S}$ group. In this functional examination, mean $2 \mathrm{D}-\mathrm{QFR}$ was $0.85 \pm 0.09$ $(\geq 0.80)$ in the NS group and $0.79 \pm 0.13(<0.80)$ in the S group, but there was no difference between the NS group and $\mathrm{S}$ group $(\mathrm{P}=0.10)$. The $3 \mathrm{D}-\mathrm{QFR}$ was significantly higher in participants with an NS strategy than in those with an S strategy $(0.86 \pm 0.08$ vs. $0.78 \pm 0.15, \mathrm{P}=0.02)$, with the former $\geq 0.80$ and the latter $<0.80$. Microcirculation resistance (MR; $\mathrm{P}=0.13)$ or anterograde blood flow $(\mathrm{P}=0.98)$ showed no significant difference (Table 8).

\section{Discussion}

In our previous study, we found no further clinical benefits with the use of DS implantation strategy compared with IS strategy for patients with STEMI and a high thrombus burden during primary PCI. However, 21 of 51 (41.2\%) participants with a DS strategy did not receive stent implantation (non-stenting strategy, NS) as a result of nonsevere stenosis in the culprit lesion and favorable epicardial blood flow without recurrent ischemic symptoms (11). 
Table 7 Comparison of IVUS information in the IRA or culprit lesion between NS and S

\begin{tabular}{|c|c|c|c|}
\hline IVUS information & NS $(n=10)$ & $S(n=15)$ & $P$ value \\
\hline MLD (mm) & $2.08 \pm 0.33$ & $2.01 \pm 0.35$ & 0.61 \\
\hline Min. lumen diameter stenosis (\%) & $44.61 \pm 11.48$ & $47.84 \pm 11.32$ & 0.49 \\
\hline $\min$ EEM CSA $\left(\mathrm{mm}^{2}\right)$ & $15.25 \pm 4.60$ & $17.05 \pm 5.27$ & 0.39 \\
\hline Plaque area $\left(\mathrm{mm}^{2}\right)$ & $10.99 \pm 3.84$ & $13.25 \pm 4.52$ & 0.21 \\
\hline Plaque burden (\%) & $70.79 \pm 6.46$ & $76.97 \pm 6.76$ & 0.03 \\
\hline Lumen area stenosis (\%) & $71.86 \pm 16.48$ & $82.50 \pm 17.56$ & 0.14 \\
\hline $\mathrm{RI}$ & $1.06 \pm 0.20$ & $1.07 \pm 0.19$ & 0.91 \\
\hline Plaque rupture (\%) & $10(100 \%)$ & $12(80 \%)$ & 0.13 \\
\hline RI negative (\%) & $2(20 \%)$ & $4(26.7 \%)$ & 0.70 \\
\hline RI positive (\%) & $4(40 \%)$ & $8(53.3 \%)$ & 0.51 \\
\hline
\end{tabular}

Data are shown as median (mean \pm SD) or $n$ (\%). Table format cited from previous study reference (11). MLD, minimum lumen diameter; EEM, external elastic membrane; CSA, cross-sectional area; min EEM CSA, minimum EEM CSA; MLA, minimum lumen area; RI, remodeling index.

Table 8 Comparison of QFR information in the IRA or culprit lesion between NS (last CAG) and S (last CAG before stenting)

\begin{tabular}{lccc}
\hline QFR information & NS $(n=17)$ & $S(n=29)$ & $P$ value \\
\hline 2D-QFR & $0.85 \pm 0.09$ & $0.79 \pm 0.13$ & 0.10 \\
3D-QFR & $0.86 \pm 0.08$ & $0.78 \pm 0.15$ & 0.02 \\
MR $(\mathrm{mmHg} \times \mathrm{s} / \mathrm{m})$ & $242.71 \pm 54.91$ & $215.92 \pm 57.06$ & 0.13 \\
Anterograde blood flow $(\mathrm{cm} / \mathrm{s})$ & $16.13 \pm 5.32$ & $16.16 \pm 5.48$ & 0.98
\end{tabular}

Table format cited from previous study reference (11). NS, non-stenting strategy; S, stenting strategy; 2D-QFR, two-dimensional QFR; 3D-QFR, three-dimensional QFR; MR, microcirculation resistance.

Among a total of 51 participants, $21(41.2 \%)$ or $30(58.8 \%)$ ultimately underwent an NS or an S strategy, respectively. Clinical or interventional characteristics at baseline were well matched between the groups, with the exception of the length of the culprit lesion $(\mathrm{P}=0.03)$, with longer lesions in patients with an $\mathrm{S}$ strategy. It has been shown that lesion length is associated with pathophysiological coronary artery ischemia, and long lesions lead to myocardial functional disorder and ischemic symptoms (12-15). Based on the evidence and our experience, we consider that in real-world procedures, physicians might prefer stent implantation in patients with STEMI who have longer mild-to-moderate target lesions.
In post hoc analysis, we set a composite of MACEs (recurrent MI, cardiac death, all-cause-mortality, HFinduced readmission, major bleeding, and target-vessel revascularization) as primary endpoints. We found that few MACEs occurred during follow-up from 1 month to 12 months, which was consistent with our previous results in patients with both DS and immediate stenting (IS) (11). Only 1 recurrent MI (3.3\%) occurred in the $\mathrm{S}$ group during hospitalization owing to acute occlusion of a non-IRA related artery, with no ischemic events among any other participants at 1- and 3-month follow-up. Until 6 or 12 months of follow-up, an extremely small proportion of cases of ischemic-induced revascularization occurred, but there 
was no difference between the two groups after statistical analysis. There are several reasons for these results: (I) all participants in this study received contemporary sufficient and effective antithrombotic treatment, which consisted of loading dose or maintenance dose dual-antiplatelet therapy, GP IIb/IIIa receptor antagonist administered perioperatively, and unfractionated heparin or bivalirudin prescribed during pPCI. Loading dose antiplatelet agents administered before the primary operation and continuation of a routine dose jointly with GP IIb/IIIa inhibitors in the first 24-72 h was shown to dissolve the thrombus and prevent ongoing thrombogenesis. During follow-up, routine use of dual-antiplatelet drugs for at least 1 year reduced the risk of new-onset of thrombotic events $(16,17)$. (II) Although plaque rupture was seen in all participants in the NS group and in most participants in the $\mathrm{S}$ group, a calcified nodule was observed in most participants in both groups. Statins were used in all participants to stabilize vulnerable lesions as well as to act as anti-inflammatory agents (18-20). On the basis of intensive antithrombotic agents and statins, the incidence of acute recurrent occlusion of the IRA clearly decreased. (III) In long-term maintenance, $\beta$-blockers, ACEIs, or ARBs were administered to most participants without contraindication. This evidence-based therapy reduced MACEs and further improved long-term outcomes (21,22). (IV) According to baseline characteristics in the NS and S groups, the mean culprit lesion stenosis rate $(49 \% \pm 26 \%$ vs. $56 \% \pm 18 \%, \mathrm{P}=0.24)$ was intermediate, with no difference between groups. All 51 participants were traced closely through outpatient services or by telephone, and the recommendations for risk-factor control were fully achieved. Effective comprehensive management of medical visits and optimal pharmacotherapy for patients with nonsevere stenosis lesions are also very important.

Few other studies have focused on whether an NS strategy could result in better or worse prognosis in patients with STEMI, with or without a high thrombus burden. In our study, we discovered that the NS strategy was not inferior to the S strategy in patients with STEMI and a high thrombus burden and non-severe stenosis lesion. A previous study of IVUS-guided treatment of patients with STEMI and a high thrombus burden defined "lowrisk" patients with STEMI as those meeting the following criteria in IVUS: MLA $\geq 4.0 \mathrm{~mm}^{2}$ or plaque burden $\leq 70 \%$ and plaque fibrous cap thickness $\geq 0.7 \mathrm{~mm}$ in the culprit lesion; high-risk patients were defined as MLA $<4.0 \mathrm{~mm}^{2}$ or plaque burden $\geq 70 \%$ and plaque fibrous cap thickness $<0.7 \mathrm{~mm}$. The researchers discovered that some "low- risk" patients with STEMI who did not require coronary stent implantation guided by IVUS did not have worse outcomes (7). The EROSION study indicated that standardized dual-antiplatelet treatment did reduce thrombus of the intermediate culprit lesion and effectively guided in optical coherence tomography (OCT) reexamination, leading to a non-inferior prognosis in patients with STEMI but without stent implantation $(8,23)$.

We considered that anatomical or morphological techniques (such as IVUS/OCT) and functional examinations (such as fraction flow reserve/QFR) could be jointly used to further assess the possibility of an NS strategy in patients with STEMI who have mild-tomoderate culprit lesions. In this post hoc analysis, IVUS testing was performed in 10 of $21(47.6 \%)$ participants from the NS group and 15 of $30(50.0 \%)$ participants from the $\mathrm{S}$ group and morphological information was collected (Table 7). We found that the MLA value was $4.27 \pm 1.02 \mathrm{~mm}^{2}\left(\geq 4.0 \mathrm{~mm}^{2}\right)$ in the NS group, and $3.80 \pm 1.32 \mathrm{~mm}^{2}\left(<4.0 \mathrm{~mm}^{2}\right)$ in the $\mathrm{S}$ group, despite no significant difference between the 2 groups $(\mathrm{P}=0.36)$. Furthermore, the plaque burden value was $70.79 \% \pm 6.46 \%$ $(\leq 70 \%)$ in the NS group and $76.97 \% \pm 6.76 \%(>70 \%)$ in the $\mathrm{S}$ group, with significant differences between the groups $(\mathrm{P}=0.03)$. Information of plaque fibrous cap thickness was unavailable in most lesions due to plaque rupture. We found that culprit lesion characteristics met the "low-risk" standard in participants from the NS group and the "highrisk" standard in patients from the $\mathrm{S}$ group, according to previous criteria (8). Further evaluation showed a smaller mean lumen area stenosis rate of $71.86 \% \pm 16.48 \%(\leq 75 \%)$ compared with $82.50 \% \pm 17.56 \%(>75 \%)$, although this did not differ between the 2 groups $(\mathrm{P}=0.14)$. Based on intraluminal IVUS examination results, an NS strategy may be an alternative for "low-risk" patients with STEMI.

The goal of re-opening the IRA and reducing stenosis of the lesion is to restore blood flow in the epicardial artery, further improving myocardial circulation and heart function. We utilized QFR analysis [a new technique that quickly and accurately computes the fractional flow reserve (FFR) value using 2- or 3D coronary artery reconstruction as well as fluid dynamics computation based on angiographic imaging (24-26)] to determine the physiological and functional values of IRA-anterograde blood flow. Both the cutoff points of 2D-QFR (computed with a single angiographic image) and 3D-QFR (computed using multiple angiographic images) were 0.80 ( $\geq 0.80$ was without ischemia and $<0.80$ was obvious ischemia). Among 
the 51 participants, QFR analysis was performed on 17 of $21(81.0 \%)$ participants in the NS group (last CAG) and 29 of $30(96.7 \%)$ participants in the S group (last CAG before stenting). We found that both 2D-QFR and 3D-QFR values were $\geq 0.80$ in the NS group, and both values were $<0.80$ in the $S$ group, with significant differences in $3 \mathrm{D}-\mathrm{QFR}(\mathrm{P}=0.02)$. According to these results, we concluded that when obvious ischemia was excluded by functional testing in patients with STEMI, the NS strategy may be an alternative.

As for the prerequisites for an NS strategy in patients with STEMI based on the results of our study, if the following conditions are met, NS might be an option: (I) ischemic symptoms completely mitigated without recurrence; (II) dynamic changes of electrocardiogram (ECG) absent; (III) anterograde blood flow restored; (IV) intraluminal examination using IVUS or OCT confirming a "low-risk" lesion; (V) physiological testing with QFR or FFR confirming obvious ischemia; and (VI) administration of standardized pharmacotherapy. Additional randomized trials are needed to further investigate the feasibility of NS strategy and to validate non-inferior outcomes using this approach.

\section{Limitations}

First, this study was a post hoc analysis of our prior observational study, which was not conducted using a randomized controlled trial design; therefore, selection bias may be present. However, a total of 51 participants received contemporary, optimal, and standardized medical therapy as well as coronary artery procedures performed by experienced physicians. Clinical and interventional baseline characteristics were comparable between participants in the 2 groups. Second, the sample size of this study was small. In our prior cohort study, we set a target for a total of 106 participants and finally recruited 245 patients with STEMI. Our post hoc analysis of 51 patients with a DS strategy made up a small proportion within our prior trial but was similar to previous studies $(27,28)$. To strengthen the robustness of our results, we introduced IVUS (as a morphological detection tool) and QFR (as a physiological and functional test) into the analysis. The consistent results in both examinations validated our conclusions. Third, although we performed follow-ups at 1, 3, 6, and 12 months that included long-term outcomes, this may be insufficient to evaluate MACEs for patients with coronary artery disease. Follow-ups up to 24 months are ongoing to assess whether NS strategy does have advantages in patients with STEMI and high thrombus burden.

\section{Conclusions}

The NS strategy did not increase the risk of MI, cardiac death, all-cause mortality, revascularization, heart failureinduced readmission, or bleeding events (MACEs). If all 3 of the following criteria are met: (I) symptoms relieved and absence of dynamic ECG changes after IRA blood flow restoration, (II) validation of non-ischemic evidence in intraluminal morphological testing (IVUS) and physiological testing (QFR), and (III) continuous standardized pharmacotherapy and risk factors are controlled, the NS strategy can be a safe alternative for patients with STEMI and a high thrombus burden. Additional research is needed to further validate the safety and effectiveness of this strategy.

\section{Acknowledgments}

We thank the staff of the Department of Cardiology at Guangdong Cardiovascular Institute, the Department of Interventional Treatment of Guangdong Provincial People's Hospital Zhuhai Hospital, and the Department of Cardiology of Guangdong Provincial Jiexi People's Hospital for their support. We thank LetPub (www.letpub.com) for its linguistic assistance during the preparation of this manuscript.

Funding: This research was funded and supported by the National Key Research and Development Program of China (2016YFC1301202).

\section{Footnote}

Reporting Checklist: The authors have completed the STROBE reporting checklist. Available at https://dx.doi. org/10.21037/apm-21-2612

Data Sharing Statement: Available at https://dx.doi. org/10.21037/apm-21-2612

Conflicts of Interest: All authors have completed the ICMJE uniform disclosure form (available at https://dx.doi. org/10.21037/apm-21-2612). All authors reported that this research was funded and supported by the National Key Research and Development Program of China (2016YFC1301202). The authors have no other conflicts of 
interest to declare.

Ethical Statement: The authors are accountable for all aspects of the work in ensuring that questions related to the accuracy or integrity of any part of this research are appropriately investigated and resolved. All participants were informed about the purpose of the study, and written informed consent was provided by all participants included in the study. All procedures performed in this study involving human participants were in accordance with the Declaration of Helsinki (as revised in 2013). This research was approved by the Ethics Committee of Guangdong Provincial People's Hospital [GDREC2018346H(R2)].

Open Access Statement: This is an Open Access article distributed in accordance with the Creative Commons Attribution-NonCommercial-NoDerivs 4.0 International License (CC BY-NC-ND 4.0), which permits the noncommercial replication and distribution of the article with the strict proviso that no changes or edits are made and the original work is properly cited (including links to both the formal publication through the relevant DOI and the license). See: https://creativecommons.org/licenses/by-nc-nd/4.0/.

\section{References}

1. Heusch G, Gersh BJ. The pathophysiology of acute myocardial infarction and strategies of protection beyond reperfusion: a continual challenge. Eur Heart J 2017;38:774-84.

2. Konijnenberg LSF, Damman P, Duncker DJ, et al. Pathophysiology and diagnosis of coronary microvascular dysfunction in ST-elevation myocardial infarction. Cardiovasc Res 2020;116:787-805.

3. Ibanez B, James S, Agewall S, et al. 2017 ESC Guidelines for the management of acute myocardial infarction in patients presenting with ST-segment elevation: The Task Force for the management of acute myocardial infarction in patients presenting with ST-segment elevation of the European Society of Cardiology (ESC). Eur Heart J 2018;39:119-77.

4. Byrne RA, Joner M, Kastrati A. Stent thrombosis and restenosis: what have we learned and where are we going? The Andreas Grüntzig Lecture ESC 2014. Eur Heart J 2015;36:3320-31.

5. Pijls NH. Acute myocardial infarction and underlying stenosis severity. Am J Cardiol 2009;103:1204-5.

6. Frøbert $\mathrm{O}$, van't Veer $\mathrm{M}$, Aarnoudse $\mathrm{W}$, et al. Acute myocardial infarction and underlying stenosis severity. Catheter Cardiovasc Interv 2007;70:958-65.

7. Wang HX, Dong PS, Li ZJ, et al. Application of Intravascular Ultrasound in the Emergency Diagnosis and Treatment of Patients with ST-Segment Elevation Myocardial Infarction. Echocardiography 2015;32:1003-8.

8. Jia H, Dai J, Hou J, et al. Effective anti-thrombotic therapy without stenting: intravascular optical coherence tomography-based management in plaque erosion (the EROSION study). Eur Heart J 2017;38:792-800.

9. Ashby DT, Dangas G, Mehran R, et al. Comparison of clinical outcomes using stents versus no stents after percutaneous coronary intervention for proximal left anterior descending versus proximal right and left circumflex coronary arteries. Am J Cardiol 2002;89:1162-6.

10. Tu S, Barbato E, Köszegi Z, et al. Fractional flow reserve calculation from 3-dimensional quantitative coronary angiography and TIMI frame count: a fast computer model to quantify the functional significance of moderately obstructed coronary arteries. JACC Cardiovasc Interv 2014;7:768-77.

11. Luo D, Hu X, Sun S, et al. The outcomes in STEMI patients with high thrombus burden treated by deferred versus immediate stent implantation in primary percutaneous coronary intervention: a prospective cohort study. Ann Transl Med 2021;9:573.

12. Iguchi $T$, Hasegawa $T$, Nishimura $S$, et al. Impact of lesion length on functional significance in intermediate coronary lesions. Clin Cardiol 2013;36:172-7.

13. López-Palop R, Carrillo P, Cordero A, et al. Effect of lesion length on functional significance of intermediate long coronary lesions. Catheter Cardiovasc Interv 2013;81:E186-94.

14. Javadzadegan A, Moshfegh A, Qian Y, et al. The relationship between coronary lesion characteristics and pathologic shear in human coronary arteries. Clin Biomech (Bristol, Avon) 2018;60:177-84.

15. Sethi A, Bahekar A, Doshi H, et al. Tirofiban use with clopidogrel and aspirin decreases adverse cardiovascular events after percutaneous coronary intervention for ST-elevation myocardial infarction: a meta-analysis of randomized trials. Can J Cardiol 2011;27:548-54.

16. Karathanos A, Lin Y, Dannenberg L, et al. Routine Glycoprotein IIb/IIIa Inhibitor Therapy in ST-Segment Elevation Myocardial Infarction: A Meta-analysis. Can J Cardiol 2019;35:1576-88.

17. Shibutani H, Fujii K, Matsumura K, et al. Differential 
influence of lesion length on fractional flow reserve in intermediate coronary lesions between each coronary artery. Catheter Cardiovasc Interv 2020;95:E168-74.

18. Baigent C, Keech A, Kearney PM, et al. Efficacy and safety of cholesterol-lowering treatment: prospective metaanalysis of data from 90,056 participants in 14 randomised trials of statins. Lancet 2005;366:1267-78.

19. Cannon CP, Braunwald E, McCabe CH, et al. Intensive versus moderate lipid lowering with statins after acute coronary syndromes. N Engl J Med 2004;350:1495-504.

20. Schwartz GG, Olsson AG, Ezekowitz MD, et al. Effects of atorvastatin on early recurrent ischemic events in acute coronary syndromes: the MIRACL study: a randomized controlled trial. JAMA 2001;285:1711-8.

21. Pfeffer MA, Greaves SC, Arnold JM, et al. Early versus delayed angiotensin-converting enzyme inhibition therapy in acute myocardial infarction. The healing and early afterload reducing therapy trial. Circulation 1997;95:2643-51.

22. Pfeffer MA, McMurray JJ, Velazquez EJ, et al. Valsartan, captopril, or both in myocardial infarction complicated by heart failure, left ventricular dysfunction, or both. N Engl J Med 2003;349:1893-906.

23. Xing L, Yamamoto E, Sugiyama T, et al. EROSION Study (Effective Anti-Thrombotic Therapy Without Stenting: Intravascular Optical Coherence Tomography-Based

Cite this article as: Luo D, Yang $\mathrm{X}, \mathrm{Hu} \mathrm{X}$, Liu J, Wang C, Ye J, Guo X, Xu S, Sun B, Liu Y, Sun S, Zhou Y, Huang Z, Zhang Y, Dong H. Non-stenting strategy is not inferior to stent implantation in patients with acute ST-segment elevated myocardial infarction and high thrombus burden and intermediate stenotic culprit lesion. Ann Palliat Med 2021;10(10):10849-10860. doi: 10.21037/apm-21-2612
Management in Plaque Erosion): A 1-Year Follow-Up Report. Circ Cardiovasc Interv 2017;10:e005860.

24. Song L, Tu S, Sun Z, et al. Quantitative flow ratioguided strategy versus angiography-guided strategy for percutaneous coronary intervention: Rationale and design of the FAVOR III China trial. Am Heart J 2020;223:72-80.

25. Tu S, Westra J, Yang J, et al. Diagnostic Accuracy of Fast Computational Approaches to Derive Fractional Flow Reserve From Diagnostic Coronary Angiography: The International Multicenter FAVOR Pilot Study. JACC Cardiovasc Interv 2016;9:2024-35.

26. Xu B, Tu S, Qiao S, et al. Diagnostic Accuracy of Angiography-Based Quantitative Flow Ratio Measurements for Online Assessment of Coronary Stenosis. J Am Coll Cardiol 2017;70:3077-87.

27. Carrick D, Oldroyd KG, McEntegart M, et al. A randomized trial of deferred stenting versus immediate stenting to prevent no- or slow-reflow in acute STsegment elevation myocardial infarction (DEFERSTEMI). J Am Coll Cardiol 2014;63:2088-98.

28. Ke D, Zhong W, Fan L, et al. Delayed versus immediate stenting for the treatment of ST-elevation acute myocardial infarction with a high thrombus burden. Coron Artery Dis 2012;23:497-506.

(English Language Editor: J. Jones) 